\title{
Modern Problems in the Surgical Treatment of Epilepsy
}

\author{
Mamytov Mitalip ${ }^{1,}$, Kadyrov Ruslan $^{1}$, Mamytova Elmira ${ }^{2}$ \\ ${ }^{1}$ Department of Neurosurgery, Kyrgyz State Medical Academy, Kyrgyzstan, Bishkek \\ ${ }^{2}$ Department of Neurology, Kyrgyz State Medical Academy, Kyrgyzstan, Bishkek
}

Email address:

mitalipmamytov@gmail.com (M. Mitalip)

${ }^{*}$ Corresponding author

\section{To cite this article:}

Mamytov Mitalip, Kadyrov Ruslan, Mamytova Elmira. Modern Problems in the Surgical Treatment of Epilepsy. Clinical Neurology and Neuroscience. Vol. 5, No. 1, 2021, pp. 5-9. doi: 10.11648/j.cnn.20210501.12

Received: February 16, 2021; Accepted: March 1, 2021; Published: March 9, 2021

\begin{abstract}
Background: The surgical treatment of epilepsy is widely recognised by neurosurgeons as the most effective method for treating patients with drug-resistant forms of the disease. Objective: To analyse the results of surgical treatments in patients with epileptic seizures, depending on the applied methods of surgery. Methods: A comprehensive clinical examination of the surgical treatments of 112 patients with various forms of drug-resistant epilepsy was carried out. Results: Hemispherectomy was performed in three patients with positive outcomes. Hippocampectomy was performed for four patients. In two patients, the seizures became medically controlled. In the other two cases, the intervals between seizures were longer and seizures tended to occur at night. Extratemporal resection of the epileptogenic focus was performed in 86 patients, $72 \%$ percent of whom became seizure-free. Six patients underwent callosotomy, five of whom were then seizure free. One patient's seizures resumed after 8 months, this case was also drug-resistant. Thirty two patients underwent multiple subpial transection and were followed-up for approximately 5 years. Excellent results were obtained in $20(62.5 \%)$ patients with seizures ceasing. Conclusions: The results of the study showed sufficient efficiency of the applied methods of surgical treatment for various forms of epilepsy.
\end{abstract}

Keywords: Drug-resistant Forms of Epilepsy, Epileptogenic Zone, Surgical Treatment of Epilepsy

\section{Introduction}

One of the most common human diseases, epilepsy, was identified at least 2500 years ago. At the time, the nature of the disease was considered supernatural and the epileptic patient themself was considered possessed by various evil spirits, each of which had its own type of seizure. Today, epilepsy has not lost its mystical character. The reason for this is the unclearness of the origin of the disease, the severity, and originality of the manifestations of a major epileptic seizure, which make an impression even on medical personnel [1-6].

This disease in our time is also one of the most common, which affects tens of millions of people in the world. Seizures in people with epilepsy are preventable with anticonvulsants. But along with this, there are many patients with epilepsy, in the treatment of which anticonvulsants do not work, i.e. these people suffer from drug-resistant forms of epilepsy. For the treatment of such patients, surgical methods of treatment are used, which are widely recognized by neurosurgeons as the most effective method of treating patients with pharmacoresistant forms of the disease.

This work analyzes the results of surgical treatment of epilepsy, using various methods of surgery, depending on the form of epilepsy. It also provides a description of our approaches to treating patients with this ailment, which have been worked out over many years of surgical treatment of epilepsy, in order to share ourr experience with doctors from the rest of the world who are also involved in the treatment of this ailment.

The first to implement a scientific approach to the interpretation of epilepsy, was the most famous physician of antiquity, Hippocrates. In the 5th century BC. Hippocrates believed that the cause of an epileptic seizure was the brain. In Hippocrates, there are references to generalised and focal seizures. In addition, there are indications of idiopathic, or primary and symptomatic, or secondary, epilepsy [5, 7].

The modern approach to epilepsy is associated with the 
works of prominent scientists of the world, such as Jackson in London, Penfield in Montreal, Davidenkov in Leningrad, Gasteau in Marseilles, and Kreindler in Bucharest. In 1861, Jackson first proposed an exhaustive theory, according to which epileptic seizures originate from an abnormal morphofunctional focus in the brain. The main clinical signs of epilepsy are convulsions of various natures with loss, or possibly without loss, of consciousness of the patient [8]. Of particular importance was the invention of the electroencephalograph by G. Berger in 1920 which marked the beginning of a new era in clinical neurophysiology and epileptology. Methodically, the development of electroencephalography (EEG) has made it possible to ascertain the presence of epileptic activity in a patient and to determine its exact focal location in the brain. Since 1959, EEG has been an indispensable tool in neurosurgery. Being the most famous neurosurgeon and Harvey Cushing's student, Wilder Penfield, during an operation for epilepsy, produced irritation with electrical current in the epileptogenic zones and adjacent areas of the cerebral cortex, thereby monitoring the effect of this irritation and receiving verbal, sensory, and motor responses. Thus, Penfield and his students used epilepsy as a model to study the functional anatomy of the human brain. For this reason, we can say that the Penfield school made a huge contribution to the surgical treatment of epilepsy. It is pertinent to note here that Penfield and Jasper pointed out that the complete removal of the hippocampus promises successful outcomes. However, in our opinion, partial hippocampectomy is more effective in the presence of incisural or hippocampal sclerosis, as confirmed by MRI. Such changes in the brain cause complex convulsive components in patients that are not amenable to drug therapy.

The first report of a callosotomy for epilepsy was reported in 1940 and described that the involvement of the corpus callosum in the tumour process was manifested in patients with generalised seizures, but as the tumour destroyed more and extent more corpus callosum, the seizures stopped [9]. On this basis, the authors proposed anterior commissurotomy for the treatment of epilepsy. Interest in callosotomy as a treatment was low until 1962, when J. Bogen reported a group of patients who underwent callosotomy as an alternative to hemispherectomy [10]. Later, callosotomy was used for epilepsy with generalised and multifocal seizures.

The multiple subpial transection method, as a new method of surgical treatment of focal epilepsy, with a detailed morphofunctional and clinical-pathogenetic rationale, was first described in 1989 [11]. It was found that the minimum volume of the cerebral cortex capable of spontaneous production and maintenance of epileptic activity is $12.5 \mathrm{~mm}^{3}$. Cortical islets wider than $5 \mathrm{~mm}$ and the horizontal joints of the cerebral tract of the same length can generate and transmit paroxysmal discharges. If two neuronal cell islets are located at a distance of $4-5 \mathrm{~mm}$ from each other, then abnormal electrical bursts become synchronous. If two similar cortical islets are at a distance of 6 or $7 \mathrm{~mm}$ from each other, the fusion of pathological discharges does not occur. Thus, it was found that paroxysmal discharges tended to become synchronous if two epileptic cortical islets were 4 $\mathrm{mm}$ apart, whereas they remained independent if their action potential was separated by a $6 \mathrm{~mm}$ cortical field distance.

\section{Methods}

The study material was the results of the surgical treatment of 112 patients with drug-resistant forms of epilepsy. The following methods were used to make the diagnosis and treat patients:

1) The neurological method with an assessment of the nature and types of seizures.

2) A diagnostic algorithm for epileptic seizures was compiled.

3) Registration and evaluation of interictal EEG

4) Magnetic resonance imaging (MRI) examination of the brain

5) Resection (anterior temporal lobectomy, hippocampectomy, hemispherectomy, or extracorporeal resection) and functional (callosotomy and multiple subpial transection) surgical methods were used.

The types of epileptic seizures were established on the basis of the classification adopted by the World League Against Epilepsy in 1989 (International league against epilepsy). In the patients' medical, endogenous and exogenous factors that could contribute to the development of epilepsy were studied. In post-traumatic epilepsy, the severity of traumatic brain injury was taken into account. In these cases, the duration between the injury and the development of epilepsy was established. According to our studies, for the majority of patients $(85 \%)$, this period did not exceed two years.

The results of surgical treatment were assessed using the Engel scale from grade I to IV: class I, no seizures $(72.3 \%)$; class II, rare seizures (14.5\%); class III, tangible improvement with a reduction in seizures $(9.8 \%)$; class IV, no tangible improvement (4.1\%) [12]. Taking into account the anamnestic data, the nature and types of epileptic seizures, we developed a diagnostic algorithm (Figure 1). The developed algorithm facilitated the selection of patients for the use of neurosurgical treatment of epilepsy.

Computed tomography (CT) and MRI were performed for all patients with epilepsy. The study involved structural neuroimaging of the brain in the axial, coronal, and sagittal planes. The CT and MRI results were compared with the clinical and EEG data, which enabled the identification of pathophysiological and structural-pathomorphological changes in the focal and diffuse nature of the brain in each particular case. These changes were of a multidisciplinary nature, aimed at determining whether the patient was a candidate for surgical treatment or whether there are risks associated with surgical intervention.

Callosotomy was performed by osteoplastic craniotomy in the frontoparietal region with subsequent interhemispheric access. After the U-shaped incision of the dura mater was made, the interhemispheric fissure was exposed. We advise aiming to preserve bridging veins and other veins. Cotton 
strips were inserted on the cortical surface of the brain, and then a spatula with a retractor was installed to allow the opening of the interhemispheric fissure. Pericallosal arteries were identified on each side. Then, they were pulled to the side with a spatula. The whiteish corpus callosum is exposed to the desired callosotomy scale. It is advisable to plan the volume of callosotomy (full or partial) prior to the start of the callosotomy surgery itself. In the centre of the operating field, on the corpus callosum, with the help of coagulation, a small hole was then formed to a sheet of a transparent septum. The callosotomy was extended back and forth to achieve the intended or desired separation of the cerebral hemispheres. After the completion of the callosotomy operation, thorough haemostasis was performed. The dura mater was sutured and the bone flap was placed in its place and strengthened to the skull, followed by layer-by-layer sutures for the soft tissue.

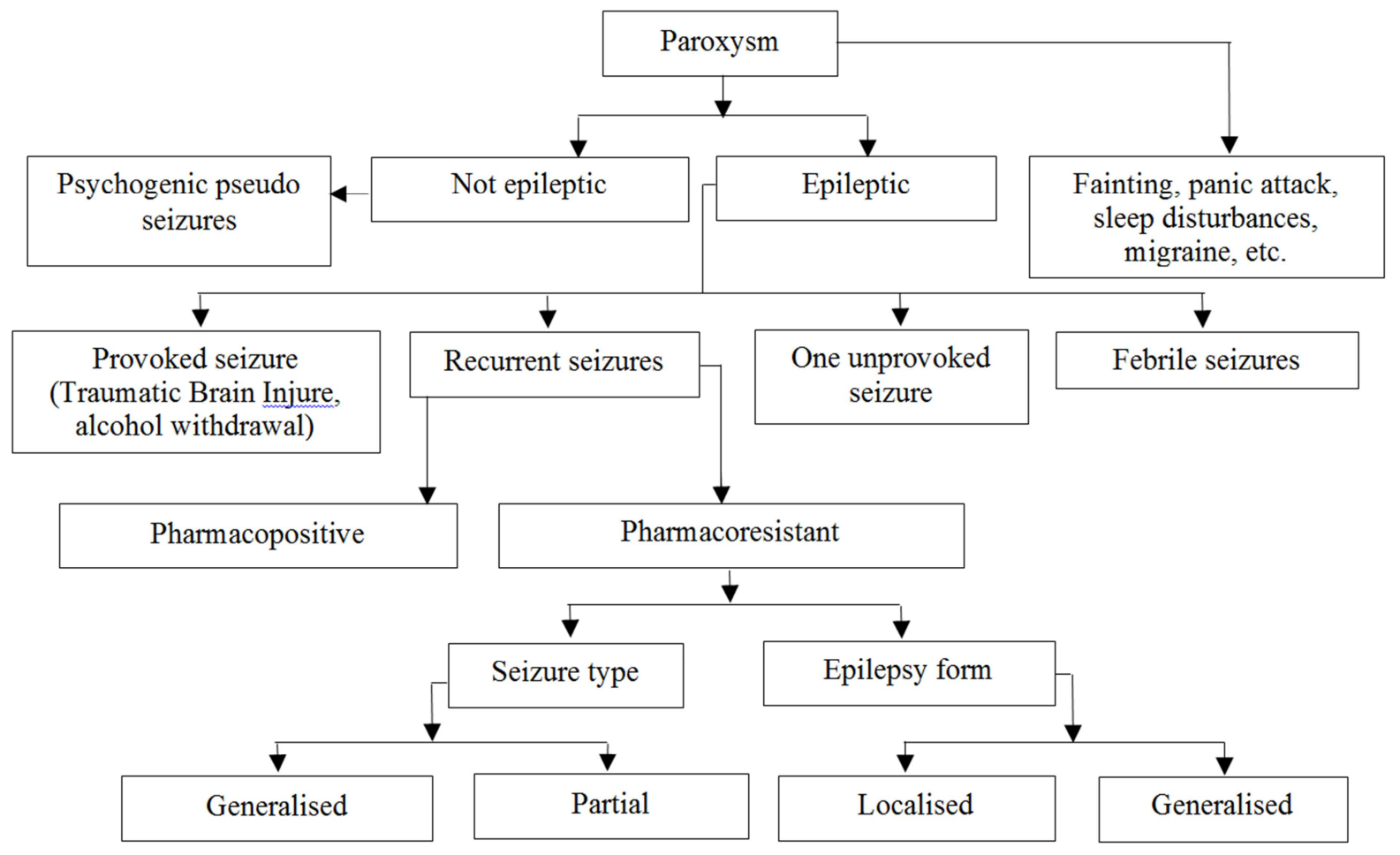

Figure 1. Diagnostic algorithm for epileptic seizures.

The multiple subpial transection method was used in 32 patients. It is known that paroxysmal discharges tend to become synchronous if two epileptic cortical islets were 4 $\mathrm{mm}$ apart, whereas they remained independent if their action potential was separated by a $6 \mathrm{~mm}$ cortical field distance. Based on the foregoing, we determined the interval of the applied dissection of multiple subpial transection (MST) as 5 $\mathrm{mm}$, which would make it possible to divide the epileptogenic zone into separate isolated cortical fields that are incapable of the generalisation of pathological electrical activity. The principle of the MST procedure itself is the dissection of horizontally oriented intracortical fibres, while preserving the majority of vertical, functionally significant neuronal fibres and penetrating pial vessels, most of which have a horizontal orientation.

For this surgery, an incision was made perpendicular to the axis of the cerebral gyrus with an interval of $5 \mathrm{~mm}$ using an original instrument designed by Morrell and a transector modified by us. The latter is a solid piece of steel wire with a handle and a hook-like tip, extending at an angle of $90^{\circ}$ and $4 \mathrm{~mm}$ long, taking into account the thickness of the cerebral cortex with a thin but not sharp tip.

\section{Results}

A total of 112 patients with drug-resistant forms of epilepsy were included in the study. The patients' ages ranged from 14 to 62 years (average age 32 years). The duration of the disease before surgery ranged from 4 months to 12 years. The diagnosis of epilepsy was made on the basis clinical, neurological, electrophysiological, and tomographic studies. All patients received anticonvulsants in the preoperative period for 6 months or more, without any effect. The patients' ages ranged from 14 to 62 years. The median duration of epilepsy was 13 years (range, 6.5 months - 17 years). The average follow-up period after surgery was 26 months (range, 7-60 months). Resection (anterior temporal lobectomy, hippocampectomy, hemispherectomy, and extracorporeal resection) and functional (callosotomy and multiple subpial transections) methods of surgical intervention were applied.

Three patients underwent anterotemporal lobectomy. After surgery, all three patients were free of seizures. The observation period after surgery was from 1.5 to 3 years. Partial hemispherectomy was also performed in five patients. In three patients, the seizures became medically controlled. In the other two cases, the intervals between seizures were 
prolonged and the attacks mainly occurred at night.

Three patients underwent partial hippocampectomy. Of the three operated patients, two experienced seizures that became less prolonged and controlled, while the third case reported longer intervals between seizures which were mainly experienced in the night. We believe that a decrease in the frequency of seizures and the transition from daytime seizures to night-time is a positive result of the surgical treatment of epilepsy. These patients were followed-up for over three years.

Extracorporeal resection was performed in 63 patients. Most epileptic seizures in these patients are caused by tumours, posttraumatic cicatricial adhesions, and cystic-degenerative processes, arteriovenous malformations, and cavernous haemangiomas, as well as cortical dysplasia, which are established on tomographic studies of the brain. The EEGs revealed characteristic epileptogenic zones near the main focus, which have a different connection with deep hippocampal formations. Focal brain lesions in these patients most likely caused the development of various epileptic seizures. There are different tactics of surgical treatment for such patients. We removed the main lesion with subsequent resection of the epileptogenic zone in a partial mode or subpial transection of the cerebral cortex. Surgical manipulation and resection volume of the epileptogenic zone were determined based on the results of corticography performed during surgery. According to the follow-up data ( $>3$ years), around $70 \%$ of operated patients had no other seizures post-surgery. Minor lesions of the brain, such as cavernomas and supratentorial cysts, as well as cicatricial adhesions, which are highly epileptogenic areas, are usually pharmacoresistant and almost always undergo resection. When these processes were extratemporal, a high degree of remission was achieved.

Callosotomy was performed in six patients. All patients had been taking anticonvulsants for over four years, but despite this, the seizures progressed. The patients took 4-6 anticonvulsants daily; however, seizures occurred almost every day. Therefore, the patients and their relatives agreed to a callosotomy operation as a last hope. After the callosotomy, the convulsions in three patients stopped completely without any neurological disorders. For two patients in the early postoperative period, split-brain syndrome was observed, which presented in the form of surdomutism (lack of speech), and a slower response to stimuli with positive pathological reflexes of oral automatism. After 1.5-2 months, these symptoms regressed, speech was restored, and seizures stopped completely. In one patient, epileptic seizures resumed after 6 months and this did not respond to drug therapy. A callosotomy with widening of the dissection in the anterior and posterior directions of the corpus callosum was then performed. Despite the repeated surgery, the patient still experienced seizures. At the same time, the interval between attacks was much greater, and the severity of the attacks was noticeably less than prior to surgical treatment.

As mentioned above, 32 patients underwent MST surgery and these patients were followed-up period for 5 years. The results of the operation showed that excellent results were obtained in 20 patients $(62.3 \%)$ and good results were achieved in 9 patients (28.1\%). Their epileptic seizures were of an accidental, episodic nature and were mainly associated with exogenous causes (against the background of colds, intoxication, emotional tension, etc.). Satisfactory results were obtained for three patients (9.8\%); their seizures persisted, but the frequency was around half of that prior to surgery.

\section{Discussion}

The surgical treatment of epilepsy is widely recognised by neurosurgeons as the most effective method for treating patients with drug-resistant forms of the disease. Despite the significant success of drug therapy, more than $35-40 \%$ of patients with epileptic seizures remain pharmacoresistant. For surgical treatment, it is expedient to establish etiological factors (traumatic, inflammatory, vascular, tumour, or other processes). In addition, it is essential to determine the presence of adherent cystic changes in the brain, cortical dysplasia, or temporal sclerosis.

Numerous studies over the past decades have shown a significant difference in the results of surgery and insufficient effectiveness with frequent relapses of seizures, depending on the methods of surgical treatment used $[5,13]$. Surgical methods of epilepsy treatment are very diverse, they can be resections or functional surgeries, including temporal or extratemporal resection, hemispherectomy, methods of separation of the hemispheres, callosotomy, multiple subpial transections, among others.

The Complete cessation of seizures within one year of surgery is observed in $55-85 \%$ of patients after resection operations, in $35-75 \%$ of patients after extratemporal localization of the epileptogenic focus, and in $45-70 \%$ of patients after hemispherectomy $[14,15]$. From the literature the benefits of these surgeries are clear, since many patients are free from seizures after surgery, the frequency of seizures is significantly reduced, or they become adequate to the environment, and the social adaptation of patients improves.

The results of this study show that success can be achieved with surgical methods of epilepsy treatment. In cases of pharmacoresistance, careful preoperative preparation with the establishment of an epileptogenic focus is required. The determination of the correct surgical treatment methods is of no small importance. For the treatment of epilepsy, there are various methods of surgery, which depends on many factors, including the localization of the epileptic focus, etiological causes of the attack, and the nature of the attack itself, among others. Our studies confirmed that the best results were obtained with the use of functional methods (callosotomy and multiple subpial transection) rather than surgical resection methods (hippocampectomy and hemispherectomy). It may be that suboptimal surgical outcomes are associated with incomplete resection of the hippocampus and partial resection of the medial parts of the temporal lobe, which are possibly associated with the epileptogenic zone [16, 17]. Surgical separation of the two hemispheres by callosotomy disrupts the synchronisation of epileptiform outbreaks between the cerebral hemispheres and, therefore, effectively reduces the severity 
and frequency of mainly generalised seizures [18].

\section{Conclusion}

Various surgical intervention methods have been used to treat patients with drug-resistant forms of epilepsy. The results of surgery depend on many factors: the duration of the disease, the frequency of seizures, the causes of seizures, and the localisation of epileptogenic zones. The results of this study show that success can be achieved with surgical methods of epilepsy treatment. The most effective surgical treatment is multiple subpial transection and subtotal callosotomy. Our studies confirmed that the best results were obtained with the use of functional methods (callosotomy and multiple subpial transection) rather than surgical resection methods (hippocampectomy and hemispherectomy).

\section{Conflict of Interest}

The authors declare that they have no competing interests.

\section{Acknowledgements}

This research did not receive any grant from the public, commercial, or not-for-profit sector funding agencies.

\section{References}

[1] Guzeva VI (2007) Epilepsy and non-epileptic paroxysmal states in children. St. Petersburg: Medical Information Agency.

[2] Penfield W, Jasper H (1954) Epilepsy and the functional anatomy of the human brain. Boston: Little, Brown \& Co.

[3] Ojemann G (1988) Surgical treatment of epilepsy. Journal of Child Neurology 3: 154-154.

[4] Bien CG, Schulze-Binhage A, Soeder BM (2006) Assessment of long-term effects of epilepsy surgery with three different reference groups. Epilepsia 11: 1865-1869.

[5] Blount JP (2017) Extratemporal resections in pediatric epilepsy surgery - an overview. Epilepsia 58 (Suppl. 1): 19-27.

[6] Alonso Vanegas MA, Lew SM, Morino M, Sarmento SA (2017) Microsurgical techniques in temporal lobe epilepsy. Epilepsia 58 (Suppl. 1): 10-18.
[7] Mukhin K, Petrukhin AS (2000) Idiopathic forms of epilepsy: systematics, diagnosis, therapy. Moscow. Art-Business-Centre.

[8] Odinak MM, Dyskin DE. Epilepsy (1997) Etiopathogenesis. Clinic. Differential diagnostics. Drug treatment. St. Petersburg: Politekhnika.

[9] Van Wagenen WP, Herren RY (1940) Surgical division of the commissural pathways in the corpus callosum: relation to spread of an epileptic attack. Archives of Neurology and Psychiatry 44: 740-759.

[10] Bogen JE, Vogel PJ (1962) Cerebral commissurotomy in man: preliminary case report. Bulletin of the Los Angeles Neurological Society 27: 169-172.

[11] Morrell F, Whisler WW, Bleck TP (1989) Multiple subpial transection: a new approach to the surgical treatment of focal epilepsy. Journal of Neurosurgery 70: 231-239.

[12] Engel J Jr, Wiebe S, French J, Sperling MR, Williamson PD, Spencer DD et al. (2003) Practice parameters: temporal lobe and localized neocortical resections for epilepsy -Report of the Quality Standards Subcommittee of the American Academy of Neurology, in association with the American Epilepsy Society and the American Association of Neurological Surgeons. Neurology 60: 538-547.

[13] Jobst BC, Cascino GD (2015) Resective epilepsy surgery for drug resistant epilepsy. JAMA 313: 285-293.

[14] Mathon B, Navarro V, Bielle F, Nguyen-Michel VH, Carpentier A, Baulac M et al. (2017) Complications after surgery for mesial temporal lobe epilepsy associated with hippocampal sclerosis. World Neurosurgery 102: 639-650. e2.

[15] Ahmedov ML, Korkmaz TS, Kemerdere R, Yeni SN, Tanriverdi T (2018) Surgical and neurological complications in temporal lobe epilepsy surgery in modern era. Surgical Neurology International 9: 134.

[16] Engel J Jr (1997) Outcome with respect to epileptic seizures. In Engel J Jr, (Ed). Surgical treatment of the epilepsies. New York: Raven Press: 553-571.

[17] Salanova V, Markand O, Worth R (2005) Temporal lobe epilepsy: analysis of failures and the role of reoperation. Acta Neurologica Scandinavica 111: 126-133.

[18] Bonilha L, Kobayashi E, Rorden C, Cendes F, Li LM (2003) Medial temporal lobe atrophy in patients with refractory temporal lobe epilepsy. Journal of Neurology, Neurosurgery, and Psychiatry 74: 1627-1630. 